Pacific Journal of Mathematic 


\section{LOCAL LIMITS AND TRIPLEABILITY}

\section{TIM BROOK}

If $A$ is an object in a category $\mathscr{A}$, the properties of $\mathscr{A} / A$ (the category of objects over $A$ ) may be considered as local properties of $\mathscr{A}$. Using 'local' in this sense, the notion of local universality is defined and some of its basic properties developed. These ideas are then applied in a brief discussion of local adjunction and local limits. Finally two local tripleability theorems are given.

The Lawvere comma category of the diagram

$$
1_{\mathscr{B}}: \mathscr{B} \longrightarrow \mathscr{B} \longleftarrow \mathscr{A}: F
$$

is denoted by $\mathscr{B} / F$, in particular $\mathscr{B} / B$ denotes the category of objects over $B$, when $B$ is an object of $\mathscr{B}$.

Given a functor $F: \mathscr{A} \rightarrow \mathscr{B}$ we define [3] $\bar{F}: \mathscr{A}^{2} \rightarrow \mathscr{B} / F$ and, for each object $A$ of $\mathscr{A}, F^{A}: \mathscr{A} \mid A \rightarrow \mathscr{\mathscr { S }} / F(A)$ by the following pullback diagrams in $\mathscr{C} \mathscr{A} \mathscr{T}:-$
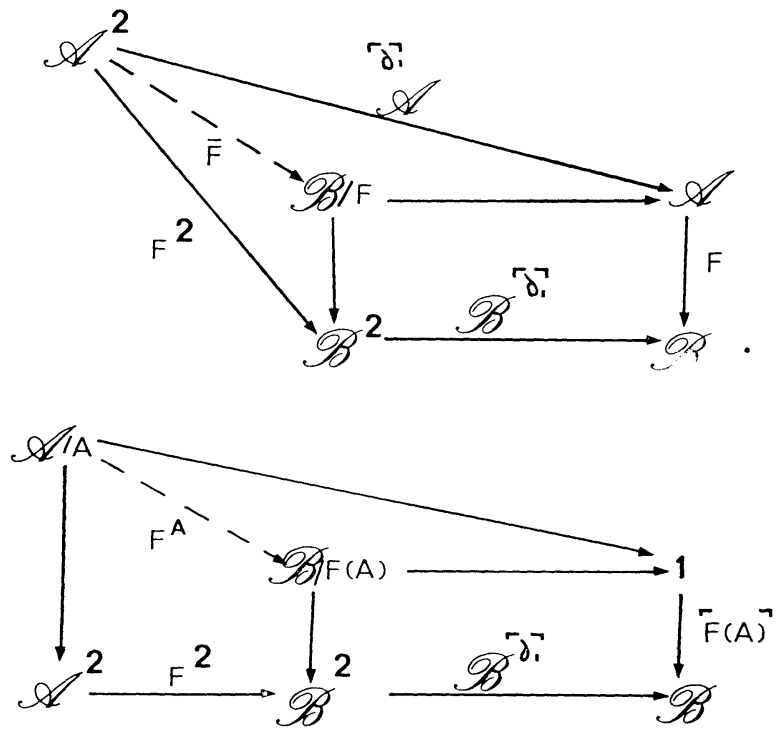

Lemma 1. For any category $\mathscr{C}$ there are isomorphisms making

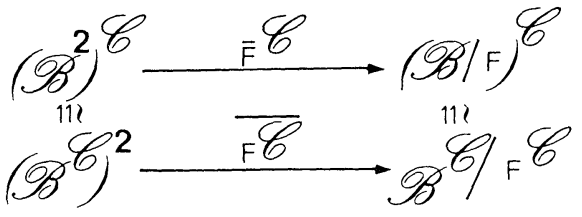

commute. 
Proof. $\mathscr{C} \mathscr{A} \mathscr{T}$ is cartesian-closed.

Let $F: \mathscr{A} \rightarrow \mathscr{B}$ be a functor and $f: B \rightarrow F(A)$ a map in $\mathscr{B}$. The pair $\left\langle f_{0}, f_{1}\right\rangle$ is a locally-universal pair (dual colocally-couniversal) from $f$ to $F$ when $f_{0}: f \rightarrow F^{A}\left(f_{1}\right) \in \mathscr{B} / F(A)$ is universal from $f$ to $F^{A}$ in the sense of MacLane [6]. Similarly a locally-couniversal pair (dual colocally-universal) from $F$ to $f$ is a pair $\left\langle f_{0}, f_{1}\right\rangle$ for which $f_{0}: F^{A}\left(f_{1}\right) \rightarrow f$ is couniversal from $F^{A}$ to $f$.

LEMMA 2. The pair $\left\langle f_{0}, f_{1}\right\rangle$ is locally-couniversal from $F$ to $f$ if and only if $\left(f_{0}, 1_{A}\right): \bar{F}\left(f_{1}\right) \rightarrow(B, f: B \rightarrow F(A), A) \in \mathscr{B} / F$ is couniversal from $\bar{F}$ to $f$.

THEOREm 1. Let $F: \mathscr{A} \rightarrow \mathscr{B}$ be a functor and $f: B \rightarrow F(A)$ a map in $\mathscr{B}$. There is a locally-couniversal pair from $F$ to $f$ if and only if there is a couniversal map from $\bar{F}$ to $(B, f: B \rightarrow F(A), A) \in \mathscr{B} \mid F$.

Proof. If $\left(f_{0}, f_{1}\right): \bar{F}\left(f_{2}\right) \rightarrow(B, f: B \rightarrow F(A), A)$ is couniversal from $\bar{F}$ to $f$, then so is $\left(f_{0}, 1_{A}\right): \bar{F}\left(f_{1} f_{2}\right) \rightarrow(B, f: B \rightarrow F(A), A)$. The result now follows by Lemma 2 .

The corresponding result for locally-universal pairs is false in general, although Kaput [3] showed that a functor $F$ is locallyadjunctable if and only if $\bar{F}$ has an adjoint.

The functor $F: \mathscr{A} \rightarrow \mathscr{B}$ is locally-adjunctable if a locally-universal pair exists for every $f: B \rightarrow F(A) \in \mathscr{B}$ and locally-coadjunctable if a locally-couniversal pair exists for every $g: F(A) \rightarrow B \in \mathscr{B}$.

Leroux [5] stated the following proposition, which is an immediate consequence of Theorem 1.

Proposition 1. A functor $F$ is locally-coadjunctable if and only if $\bar{F}$ has a coadjoint.

From which, by Lemma 1, we obtain

Proposition 2. If a functor $F: \mathscr{A} \rightarrow \mathscr{B}$, is locally-coadjunctable then so is $F^{\mathscr{C}}: \mathscr{A}^{\mathscr{C}} \rightarrow \mathscr{B}$ for any category $\mathscr{C}$.

THEOREM 2. Let $F: \mathscr{A} \rightarrow \mathscr{B}$ be a functor and $f: B \rightarrow F(A)$, $c: F\left(A^{\prime}\right) \rightarrow B, c^{\prime}: F\left(A^{\prime \prime}\right) \rightarrow F(A)$ maps in $\mathscr{B}$ with $c^{\prime}$ couniversal from $F$ to $F(A)$. By couniversality there are (unique) maps $s, t$ for which $c^{\prime} F(s)=1_{F^{\prime}(A)}$ and $c^{\prime} F(t)=f c$.

(A) Suppose $c$ is couniversal from $F$ to $B$. The diagram $s: A \rightarrow A^{\prime \prime} \leftarrow A^{\prime}: t$ has a pullback in $\mathscr{A}$ if and only if there is a locally-counivearsal pair from $F$ to $f$. 
(B) Suppose $c^{\prime}$ is an identity map. The map c is couniversal from $F$ to $B$ if and only if $\langle c, t\rangle$ is locally-couniversal from $F$ to $f$.

Proof. (A) If

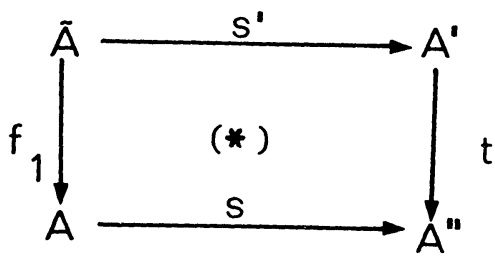

is a pullback in $\mathscr{A}$, then $\left\langle c F\left(s^{\prime}\right), f_{1}\right\rangle$ is locally-couniversal from $F$ to $f$. Conversely, if $\left\langle f_{0}, f_{1}\right\rangle$ is locally-couniversal from $F$ to $f$ then $\left(^{*}\right)$ is a pullback where $s^{\prime}$ is the unique map for which $c F\left(s^{\prime}\right)=f_{0}$.

(B) Suppose $\langle c, t\rangle$ is locally-couniversal from $F$ to $f$. For any $g: F(\widetilde{A}) \rightarrow B$, by the couniversality of $1_{F(A)}$, there is a unique $h$ for which $F(h)=f g$. Thus there is a unique $p$ for which (i) $t p=h$ and (ii) $c F(p)=g$. Again by the couniversality of $1_{F(A)}$, (ii) implies (i) and so $p$ is unique in satisfying (ii).

The converse follows directly from (A).

Corollary 1. (Leroux) If a functor $F: \mathscr{A} \rightarrow \mathscr{B}$ has a coadjoint and $\mathscr{A}$ has pullbacks then $F$ is locally-coadjunctable.

Let $\Delta: \mathscr{C} \rightarrow \mathscr{C}^{\mathscr{X}}$ be the canonical embedding. If $D: \mathscr{X} \rightarrow \mathscr{C}$ is a functor and $\rho: D \rightarrow \Delta(X) \in \mathscr{C}^{x}$, then a locally-universal pair from $\rho$ to $\Delta$ is called a local colimit for $D$ at $\rho$ and a locally-couniversal pair from $\Delta$ to $\rho$ is called a local limit for $D$ at $\rho$.

Corollary 2. (Folklore) When $\mathscr{Z}$ is a connected category, $D$ has a limit if and only if it has a local limit at $\rho$.

The definitions of local limit and colimit are the obvious ones to make (given the definition of local universality) but they are essentially renameings of limits and colimits in $\mathscr{C} / X$. Thus the standard theorems on limits, colimits and adjointness have local counterparts; moreover, in most cases the local result is a trivial corollary of the global. Propositions 3,4,5, and 6 are immediate consequences of this observation.

Proposition 3. If a functor is locally-adjunctable then it preserves $\mathscr{X}$-limits for any connected category $\mathscr{X}$. In particular it preserves pullbacks and equalizers. 
Proposition 4. If a functor is locally-coadjunctable it commutes with colimits.

A functor $U: \mathscr{B} \rightarrow \mathscr{A}$ is locally-tripleable if $U^{B}: \mathscr{B} / B \rightarrow \mathscr{A} / U(B)$ is tripleable for every object $B$ of $\mathscr{B}$.

Proposition 5. If a functor is locally-tripleable then it preserves and reflects $\mathscr{Z}$-limits for any connected category $\mathscr{X}$.

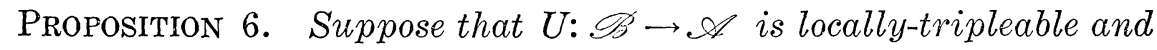
that $\mathscr{A}$ and $\mathscr{B}$ have finite products. If $U$ preserves $\mathscr{P}$-colimits then it reflects $\mathscr{P}$-colimits. (U reflects the colimits that it preserves.)

For each map $f: X \rightarrow Y \in \mathscr{C}$ the functor $f^{\prime}: \mathscr{C} \mid X \rightarrow \mathscr{C} / Y$ defined by $f^{\prime}\left(X^{\prime} \rightarrow X\right)=\left(X^{\prime} \rightarrow X \rightarrow Y\right)$, and the forgetful functor $\mathscr{C} \mid X \rightarrow \mathscr{C}$ are trivially locally-tripleable for any category $\mathscr{C}$. It is also clear that every full locally-reflective subcategory has a locally-tripleable inclusion functor. The following theorem serves to show that every tripleable functor is locally-tripleable and also that the category of fields is locally-tripleable over the category of sets.

THEOREM 3. Let $U: \mathscr{B} \rightarrow \mathscr{A}$ be a locally-adjunctable functor. $U$ is locally-tripleable if and only if

(i) U reflects coequalizers of cobounded U-contractible pairs

(ii) $\mathscr{B}$ has coequalizers of cobounded U-contractible pairs

(iii) $U$ preserves coequalizers of U-contractible pairs. (Here a cobound for a pair $d_{0}, d_{1}: X \rightarrow Y \in \mathscr{B}$ is a map $b: Y \rightarrow B \in \mathscr{B}$ for which $b d_{0}=b d_{1}$.)

The proof relies heavily on Beck's tripleability theorem [1].

Proof. If $d_{0}, d_{1}:(X \rightarrow B) \rightarrow(Y \rightarrow B) \in \mathscr{B} / B$ is a $U^{B}$-contractible pair, then $d_{0}, d_{1}: X \rightarrow Y \in \mathscr{B}$ is clearly a cobounded $U$-contractible pair. Thus (i), (ii), and (iii) are together sufficient for local tripleability. Conversely, suppose $U$ is locally-tripleable and $d_{0}, d_{1}: X \rightarrow Y \in \mathscr{B}$ is a $U$-contractible pair.

(i) Suppose that $U(d)$ is a coequalizer of $U\left(d_{0}\right)$ and $U\left(d_{1}\right)$, and thus a contractible coequalizer. For any cobound $b: Y \rightarrow B$,

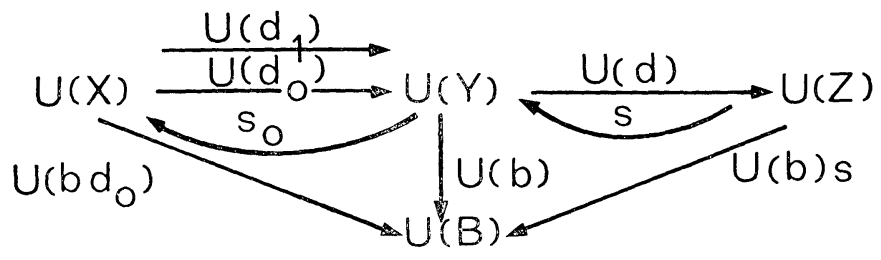


is a contractible coequalizer diagram in $\mathscr{A} / U(B)$. The category $\mathscr{B} / B$ has and $U^{B}$ preserves coequalizers of $U^{B}$-contractible pairs so, without loss of generality, $U(b) s$ may be taken to be $U(z)$ for some $z: Z \rightarrow B \in \mathscr{B}$ for which

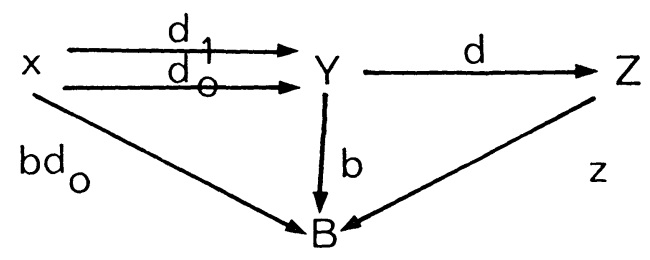

is a coequalizer diagram in $\mathscr{B} / B$.

Thus the existence of a cobound implies that $d d_{0}=d d_{1}$, and the remaining properties of a coequalizer follow as $b:(Y \rightarrow B) \rightarrow(B \rightarrow B) \epsilon$ $\mathscr{B} / B$.

(ii) If the $U$-contractible pair $d_{0}, d_{1}: X \rightarrow Y$ has a cobound $b: Y \rightarrow B$ then $d_{0}, d_{1}:\left(X \stackrel{b d_{0}}{\longrightarrow} B\right) \rightarrow(Y \stackrel{b}{\rightarrow} B)$ is a $U^{B}$-contractible pair and thus has a coequalizer, $c:(Y \rightarrow B) \rightarrow(C \rightarrow B) \in \mathscr{B} / B$. The map $U^{B}(c)$ is a coequalizer of $U^{B}\left(d_{0}\right)$ and $U^{B}\left(d_{1}\right)$ and thus a contractible coequalizer. Therefore $U(c)$ is a contractible coequalizer of $U\left(d_{0}\right)$ and $U\left(d_{1}\right)$. By (i) $c$ is a coequalizer of $d_{0}$ and $d_{1}$.

(iii) The preceding construction also serves to prove (iii).

By the same reasoning the following local version of Duskin's tripleability theorem [2] may be obtained.

THEOREM 4. Let $U: \mathscr{B} \rightarrow \mathscr{A}$ be a locally-adjunctable functor and suppose that $\mathscr{A}$ has kernel pairs. The functor $U$ is locallytripleable if and only if pairs

(i) U reflects coequalizers of cobounded U-contractible equivalence

(ii) $U$ preserves coequalizers of $U$-contractible equivalence pairs pairs

(iii) $\mathscr{B}$ has coequalizers of cobounded U-contractible equivalence

(iv) $\mathscr{B}$ has kernel pairs.

The author is indebted to Dr. S. A. Huq for suggesting this problem.

\section{REFERENCES}

1. J. Beck, untitled mimeograph, Cornell, 1966.

2. J. Duskin, Variations on Beck's Tripleability Criterion, in Reports M. W. C. S. III, Springer Lecture Notes in Mathematics, 106 (1969), 74-129.

3. J. J. Kaput, Locally adjunctable functors, Illinois J. Math., 16 (1972), 86-94. 
4. F.W. V. Lawvere, The Category of Categories as a Foundation for Mathematics, in Proc. C. O. C. A. (La Jolla, 1965). Springer, Berlin, (1966), 1-20.

5. P. Leroux, Structures et Sémantique Abstraites, in Reports M. W. C. S. V, Springer Lecture Notes in Mathematics, 195 (1971), 154-194.

6. S. MacLane, Categories for the Working Mathematician, Springer, New York, 1971.

Received November 3, 1972 and in revised form July 7, 1973.

Institute of Advanced Studies

AUSTRALIAN NATIONAL UNIVERSITY

Canberra, ACT 


\section{PACIFIC JOURNAL OF MATHEMATICS}

\section{EDITORS}

RICHARD ARENS (Managing Editor) University of California

Los Angeles, California 90024

R. A. BeaUmont

University of Washington Seattle, Washington 98105
J. DUGUNDJI*

Department of Mathematics University of Southern California Los Angeles, California 90007

D. Gilbarg and J. Milgram Stanford University

Stanford, California 94305

\section{ASSOCIATE EDITORS}

E. F. BECKENBACH

B. H. NeUmanN

F. WOLF

K. YoSHIDA

\section{SUPPORTING INSTITUTIONS}

\section{UNIVERSITY OF BRITISH COLUMBIA CALIFORNIA INSTITUTE OF TECHNOLOGY UNIVERSITY OF CA.LIFORNIA MONTANA STATE UNIVERSITY UNIVERSITY OF NEVADA NEW MEXICO STATE UNIVERSITY OREGON STATE UNIVERSITY UNIVERSITY OF OREGON OSAKA UNIVERSITY}

UNIVERSITY OF SOUTHERN CALIFORNIA STANFORD UNIVERSITY UNIVERSITY OF TOKYO UNIVERSITY OF UTAH WASHINGTON STATE UNIVERSITY UNIVERSITY OF WASHINGTON AMERICAN MATHEMATICAL SOCIETY NAVAL WEAPONS CENTER

* C. R. DePrima California Institute of Technology, Pasadena, CA 91109, will replace J. Dugundji until August 1974. 


\section{Pacific Journal of Mathematics}

\section{Vol. 50, No. $1 \quad$ September, 1974}

Gail Atneosen, Sierpinski curves in finite 2-complexes.............. 1

Bruce Alan Barnes, Representations of $B^{*}$-algebras on Banach spaces .... 7

George Benke, On the hypergroup structure of central $\Lambda(p)$ sets ....... 19

Carlos R. Borges, Absolute extensor spaces: a correction and an

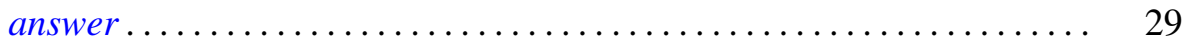

Tim G. Brook, Local limits and tripleability .................. 31

Philip Throop Church and James Timourian, Real analytic open maps .... 37

Timothy V. Fossum, The center of a simple algebra ............... 43

Richard Freiman, Homeomorphisms of long circles without periodic

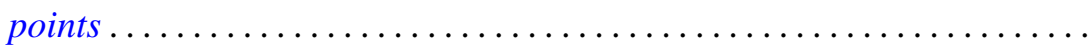

B. E. Fullbright, Intersectional properties of certain families of compact

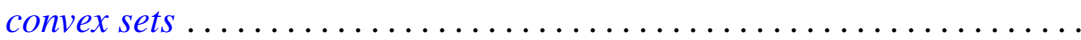

Harvey Charles Greenwald, Lipschitz spaces on the surface of the unit

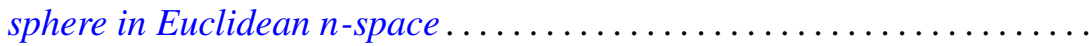

Herbert Paul Halpern, Open projections and Borel structures for

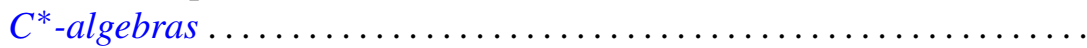

Frederic Timothy Howard, The numer of multinomial coefficients divisible

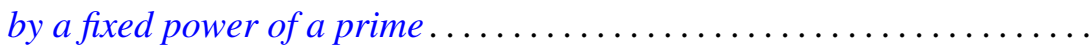

Lawrence Stanislaus Husch, Jr. and Ping-Fun Lam, Homeomorphisms of manifolds with zero-dimensional sets of nonwandering points........ 109

Joseph Edmund Kist, Two characterizations of commutative Baer rings ...

Lynn McLinden, An extension of Fenchel's duality theorem to saddle functions and dual minimax problems ................

Leo Sario and Cecilia Wang, Counterexamples in the biharmonic classification of Riemannian 2-manifolds...

Saharon Shelah, The Hanf number of omitting complete types ...

Richard Staum, The algebra of bounded continuous functions into a

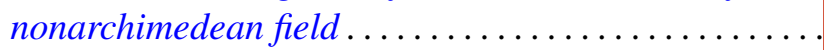

James DeWitt Stein, Some aspects of automatic continuity ..

Tommy Kay Teague, On the Engel margin

John Griggs Thompson, Nonsolvable finite groups all of whose local subgroups are solvable, $V \ldots \ldots \ldots \ldots \ldots \ldots \ldots \ldots$

Kung-Wei Yang, Isomorphisms of group extensions 